UDC $547.316+547.97$

\author{
S.A. Varenichenko ${ }^{a,}{ }^{*}$, O.K. Farat ${ }^{a}$, A.V. Mazepa ${ }^{b}$, V.I. Markov ${ }^{a}$
}

\title{
SYNTHESIS OF NEW SCHIFF BASES BASED ON FORMYL DERIVATIVES OF XANTHENES
}

\author{
${ }^{a}$ Ukrainian State University of Chemical Technology, Dnipro, Ukraine \\ ${ }^{b}$ A.V. Bogatsky Physico-Chemical Institute of the National Academy of Sciences of Ukraine, Odessa, \\ Ukraine
}

\begin{abstract}
New Schiff bases were synthesized by the reaction of tetracyclic formyl derivatives of xanthenes with aromatic amines in the presence of a vapor of acetic acid drops. The structures of these compounds were investigated by FTIR, NMR spectroscopies and massspectrometric technique. Formyl xanthenes showed absorption and emission in the range of 424-435 nm and 524-559 nm, respectively, in solvents with different polarities. Initial formyl xanthenes were characterized by a suficiently high Stokes shift of 100-130 nm and a moderate fluorescence quantum yield. The prepared Schiff bases exhibit no fluorescence, with the exception of two products with antranilic acid. These compounds show weakly structured absorption spectra with maxima at $527 \mathrm{~nm}$ and $532 \mathrm{~nm}$. Luminescence maxima were found in the range of 582 and $598 \mathrm{~nm}$; as a result, the prepared compounds are characterized by low fluorescence quantum yields in the range of $0.01-0.02$ in acetonitrile solution and the Stokes shift of $55 \mathrm{~nm}$ and $65 \mathrm{~nm}$.
\end{abstract}

Keywords: formyl xanthenes; Schiff bases; aromatic amines; fluorescence; Stokes shift.

DOI: $10.32434 / 0321-4095-2019-126-5-22-26$

\section{Introduction}

The synthesis and characterization of $\pi$-conjugated organic compounds is nowadays of great interest due to their widespread use in various branches of technology, for example, for molecular conductors, liquid crystals, electronic and optoelectronic devices [1-3].

Schiff bases are widely used both in medicine [4] and in information technology [5]. About 500 scientific papers have been published in recent years on this topic [6,7]. Azomethines are used as pigments and dyes, catalysts and intermediates in organic synthesis, in analytical chemistry and as polymer stabilizers. There are examples of their application for the extraction of metals and for the production of heat-resistant chelate polymers [8] as well as complexes with metal ions [9]. Azomethine dyes are used as components for paints in synthetic fibers production.

Conjugated polymer Schiff bases, that is polymers with a spatially extended $\pi$-electron system, have unique physical properties that are inaccessible to many other polymers. For example, these are their thermal stability, liquid crystal properties [10], fiber- forming ability, nonlinear optical and luminescent properties, electrical conductivity and the ability to chelate. In addition, polyimines synthesized from aromatic amines and aldehydes can be used as semiconductors.

Thus the synthesis of new azomethines and the study of their properties is one of the most urgent problems of modern organic synthesis.

\section{Results and discussion}

Previously, in the course of studying the rearrangement of geminal azines under the action of a formylating agent, we prepared 4,5-diformyl2,3,6,7,8,10-hexahydroacridine-8a- $(1 H)$-carbonitrile [11] and 2-methyl-4-oxo-1,5-diazaspiro[5.5]undec2-ene-3-carbaldehyde [12], in which the aldehyde group was coupled with an electron-donor substituent. Despite the reduced reactivity of the aldehyde group, Schiff bases were obtained by the reaction with various amines $[13,14]$.

Similar transformations were studied in respect to the rearrangement of geminal 1,3-naphthoxazines. The interaction of the starting compounds with the formylating agent gave rise to the formation of the expected tetracyclic formyl derivatives of xanthenes 
$1 \mathrm{a}, \mathrm{b}$ (Scheme). The obtained aldehydes 1a,b possessed luminescence in the yellow-green spectral region in organic solvents. The synthesized dyes $1 \mathrm{a}$, $\mathrm{b}$ showed absorption and emission in the range of 424-435 $\mathrm{nm}$ and 524-559 $\mathrm{nm}$, respectively, in solvents with different polarities. So, the compounds $1 \mathrm{a}, \mathrm{b}$ were characterized by suficiently large Stokes shift of $100-130 \mathrm{~nm}$ and a moderate fluorescence quantum yield [15].

The presence of formyl groups in compounds $1 \mathrm{a}, \mathrm{b}$ allowed us to carry out a number of modifications. In spite of the lower electrophilicity of the formyl groups due to conjugation with the electron-donating oxygen atom, compounds $1 \mathrm{a}, \mathrm{b}$ readily react with aromatic amines. Schiff bases 2 a$\mathrm{g}$ were synthesized in the reaction with an equimolar amount of aromatic amines when heated for 10-15 minutes in the presence of a vapor of acetic acid drops.
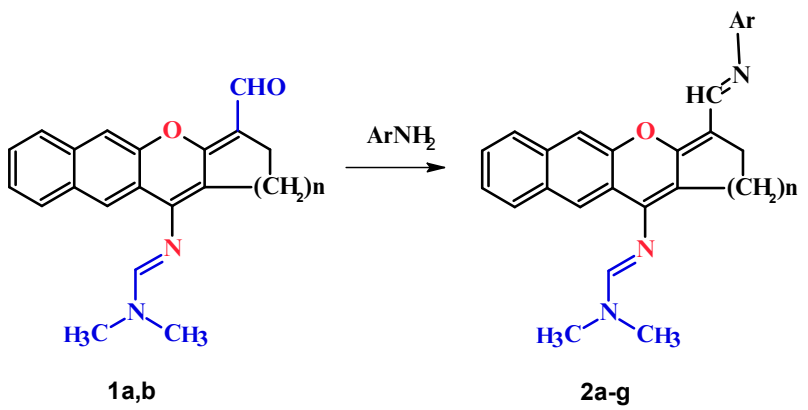

2: $\mathrm{a}-\mathrm{n}=2, \mathrm{R}_{1}=\mathrm{Ph} ; \mathrm{b}-\mathrm{n}=2, \mathrm{R}_{1}=2-\mathrm{CO}_{2} \mathrm{H}-\mathrm{C}_{6} \mathrm{H}_{4} ; \mathrm{c}-\mathrm{n}=2$, $\mathrm{R}_{1}=2$-aminopyrimidine; $\mathrm{d}-\mathrm{n}=2, \mathrm{R}_{1}=3-\mathrm{OH}-\mathrm{C}_{6} \mathrm{H}_{4} ; \mathrm{e}-\mathrm{n}=2$,

$\mathrm{R}_{1}=3-\mathrm{NO}_{2}-\mathrm{C}_{6} \mathrm{H}_{4} ; \mathrm{f}-\mathrm{n}=2, \mathrm{R}_{1}=2-\mathrm{CH}_{3}-5-\mathrm{NO}_{2}-\mathrm{C}_{6} \mathrm{H}_{3}$; $\mathrm{g}-\mathrm{n}=1, \mathrm{R}_{1}=2-\mathrm{CO}_{2} \mathrm{H}-\mathrm{C}_{6} \mathrm{H}_{4}$

In the ${ }^{1} \mathrm{H}$ NMR spectra of the products $2 \mathrm{a}-\mathrm{g}$, the proton signal of the azomethine group $(\mathrm{CH}=\mathrm{NR})$ was registered in the region of 8.86-9.6 ppm, the carbon signal of this group was located in the region of $155.0-170.3 \mathrm{ppm}$. The characteristic proton signal of the amidine fragment $\mathrm{CH}-\mathrm{N}(\mathrm{Me})_{2}$ ) corresponded to a chemical shift of $7.88-8.58 \mathrm{ppm}$. The vibrations of the $\mathrm{C}=\mathrm{N}$ group in the IR spectrum corresponded to the absorption band of $1634-1645 \mathrm{~cm}^{-1}$.

When extending the conjugated chain, the capability of luminescence was retained only for the Schiff bases $2 \mathrm{~b}$ and $2 \mathrm{~g}$. Absorption and emission spectra of the compounds $2 \mathrm{~b}$ and $2 \mathrm{~g}$ were recorded in acetonitrile for $2.5 \cdot 10^{-5} \mathrm{~mol} \mathrm{~L}^{-1}$ solutions. These compounds had weakly structured absorption spectra with maxima at 527 and $532 \mathrm{~nm}$. Luminescence maxima were found in the range of 582 and $598 \mathrm{~nm}$, so compounds $2 \mathrm{~b}$ and $2 \mathrm{~g}$ were characterized by a low fluorescence quantum yield and Stokes shifts of
55 and $65 \mathrm{~nm}$.

To conclude, new Schiff bases have been synthesized based on formyl derivatives of xanthenes in this work. The structures of these compounds have been fully characterized by FT-IR, ${ }^{1} \mathrm{H}$ NMR, ${ }^{13} \mathrm{C}$ NMR and mass spectral data. Unlike the starting aldehydes, only Schiff bases with anthranilic acid demonstrated noticeable fluorescence in a yellow part of the spectra $(2-\{[(1 E)-(12-\{[(1 E)$-(dimethylamino)methylene]amino\}-2,3-dihydro- $1 H$-benzo[b]xanten4-yl\}methylene]amino\}benzoic acid and 2-\{[(1E)(11-\{[(1E)-(dimethylamino)-methylene $]$ amino $\}-1,2-$ dihydrobenzo $[g]$-cyclopenta $[b]$ chromen-3yl)methylene]amino\}-benzoic acid). The prepared derivatives of azomethine can be used as molecular building blocks for organic synthesis.

\section{Experimental}

The ${ }^{1} \mathrm{H}$ NMR and ${ }^{13} \mathrm{C}$ NMR spectra were recorded using a Bruker Avance II 400 instrument (400.13 MHz and $100.62 \mathrm{MHz}$ for ${ }^{1} \mathrm{H}$ and ${ }^{13} \mathrm{C}$, respectively) in DMSO- $d_{6}$ or DMSO- $d_{6} / \mathrm{CF}_{3} \mathrm{CO}_{2} \mathrm{D}$ with $\mathrm{Me}_{4} \mathrm{Si}$ as internal standard. The FTIR spectra were recorded in $\mathrm{KBr}$ pellets using a Spectrum one (PerkinElmer) FT-IR spectrometer. The FAB spectra were by a VG 7070 spectrometer. Desorption of the ions from the solution of the samples in metanitrobenzyl alcohol was realized with a beam of argon atoms with energy $8 \mathrm{keV}$. Elemental analysis was performed using a LECO CHN-900 instrument. Excitation and emission luminescence spectra were registered by means of a Hitachi F-7000 spectrophotometer for acetonitrile solutions placed in a standard quartz cuvette with an optical path length $10 \mathrm{~mm}, 90^{\circ}$ geometry. Melting points were determined using an Electrothermal 9100 Digital Melting Point apparatus. The reactions and the purity of the obtained compounds were monitored by TLC on Merck Silicagel $60 \mathrm{~F}-254$ plates with 10:1 $\mathrm{CHCl}_{3}$ / $i$ - $\mathrm{PrOH}$ as eluent.

The synthesis of Schiff bases (general method)

The aldehyde $1 \mathrm{a}, \mathrm{b}(3.8 \mathrm{mmol})$ was dissolved in DMF $(5 \mathrm{~mL})$, than $3.8 \mathrm{mmol}$ of aromatic amine and a few drops of HOAc were added. The reaction solution was refluxed for $10 \mathrm{~min}$ and left off at room temperature for $12 \mathrm{~h}$. The reaction mixture was diluted with a small amount of water and crystallized for $1 \mathrm{~h}$. The formed precipitate was collected by filtration and purified by recrystallization from $\mathrm{CH}_{3} \mathrm{CN}$.

$N, N$-dimethyl-N-\{4-[(E)-(phenylimino)methyl]2,3-dihydro-1H-benzo[b]xanten-12-yl\}imidoformamide (2a):

Red powder, yield $78 \%, \mathrm{mp} 218-220^{\circ} \mathrm{C} .{ }^{1} \mathrm{H}$ NMR $\left(400 \mathrm{MHz}, \mathrm{DMSO}-d_{6}\right), \delta, \operatorname{ppm}(J, \mathrm{~Hz}): 8.89$ 
$(1 \mathrm{H}, \mathrm{s}, \mathrm{CH}=\mathrm{N}), 7.88\left(1 \mathrm{H}, \mathrm{s}, \mathrm{N}=\mathrm{CHN}(\mathrm{Me})_{2}\right), 7.87-$ 7.85 (2H, m, H Ar), 7.76-7.74 (1H, m, H Ar), 7.68 $(1 \mathrm{H}, \mathrm{s}, \mathrm{H} \mathrm{Ar}), 7.54\left(1 \mathrm{H}, \mathrm{s}, \mathrm{N}=\mathrm{CH}-\mathrm{N}\left(\mathrm{CH}_{3}\right)_{2}\right), 7.42$ (1H, m, H Ar), 7.36-7.34 (2H, m, H Ar), 7.15$7.13(3 \mathrm{H}, \mathrm{m}, \mathrm{H} \mathrm{Ar}), 3.06\left(6 \mathrm{H}, \mathrm{s}, 2 \mathrm{CH}_{3}\right), 2.59-2.55$ $\left(4 \mathrm{H}, \mathrm{m}, 2 \mathrm{CH}_{2}\right), 1.67\left(2 \mathrm{H}, \mathrm{m}, \mathrm{CH}_{2}\right) \cdot{ }^{13} \mathrm{C}$ NMR $(100$ $\left.\mathrm{MHz}, \mathrm{DMSO}-d_{6}\right), \delta$, ppm: $155.3(\mathrm{CH}=\mathrm{N}), 155.2$, $154.5,153.4,149.6,142.9,133.6,129.4,128.9,127.9$, $126.7,126.3,124.5,124.3,122.5,122.0,120.7,112.9$, 110.1, 109.6, 33.8, 24.9, 23.1, 20.6. FT-IR $(\mathrm{KBr}$ pellets, $\left.v, \mathrm{~cm}^{-1}\right)$ : 2926 (aliphatic $\left.\mathrm{C}-\mathrm{H}\right), 1636(\mathrm{C}=\mathrm{N})$. MS (FAB), $m / z\left(I_{\text {rel, }} \%\right): 408[\mathrm{M}+\mathrm{H}]^{+}(100)$. Calculated for $\mathrm{C}_{27} \mathrm{H}_{25} \mathrm{~N}_{3} \mathrm{O}(\%)$ : C, 79.58; $\mathrm{H}, 6.18$; $\mathrm{N}, 10.31$. Found $(\%)$ : C, 79.46; H, 6.29; N, 10.42 .

$2-\{[(1 E)-(12-\{[(1 E)-($ dimethylamino $)$ methylene]amino\}-2,3-dihydro-1H-benzo[b]xanten-4yl\}methylene Jamino\}benzoic acid (2b):

Purple powder, yield $86 \%, \mathrm{mp} 234-235^{\circ} \mathrm{C} .{ }^{1} \mathrm{H}$ NMR (400 MHz, DMSO- $\left.d_{6}\right), \delta, \operatorname{ppm}(J, \mathrm{~Hz}): 9.06$ $(1 \mathrm{H}, \mathrm{s}, \mathrm{CH}=\mathrm{N}), 8.24\left(1 \mathrm{H}, \mathrm{s}, \mathrm{N}=\mathrm{CHN}(\mathrm{Me})_{2}\right), 8.02-$ 8.0 (4H, m, H Ar), 7.91-7.81 (2H, m, H Ar), 7.527.44 (3H, m, H Ar), 7.19-7.17 (1H, m, H Ar), 3.15 $\left(6 \mathrm{H}, \mathrm{s}, 2 \mathrm{CH}_{3}\right), 2.64-2.59\left(2 \mathrm{H}, \mathrm{m}, \mathrm{CH}_{2}\right), 2.50(2 \mathrm{H}$, $\mathrm{m}, \mathrm{CH}_{2}$ overlapped with DMSO signals), 1.75-1.72 $\left(2 \mathrm{H}, \mathrm{m}, \mathrm{CH}_{2}\right) \cdot{ }^{13} \mathrm{C}$ NMR $(100 \mathrm{MHz}$, TFA- $d), \delta$, ppm: $175.2\left(\mathrm{CO}_{2} \mathrm{H}\right), 171.8(\underline{\mathrm{CH}}=\mathrm{N}), 158.2,152.3$, $150.8,142.6,141.3,139.2,138.6,135.9,133.8,133.2$, $131.0,130.4,129.7,126.8,126.7,119.3,118.9,116.4$, $112.3,46.9,39.5,27.0,23.9,21.2$. FT-IR (KBr pellets, $\left.v, \mathrm{~cm}^{-1}\right): 3420(\mathrm{O}-\mathrm{H}), 2925$ (aliphatic $\mathrm{C}-$ $\mathrm{H}), 1670(\mathrm{C}=\mathrm{O}), 1641(\mathrm{C}=\mathrm{N})$. MS $(\mathrm{FAB}), m / z\left(I_{\text {rel, }}\right.$ $\%): 452[\mathrm{M}+\mathrm{H}]^{+}(100)$. Calculated for $\mathrm{C}_{28} \mathrm{H}_{25} \mathrm{~N}_{3} \mathrm{O}_{3}$ (\%): C, 74.48; H, 5.58; N, 9.31. Found (\%): C, 74.56; H, 5.43; N, 9.22.

$N, N$-dimethyl-N-\{4-[(E)-(pyrimidin-2ylimino)methyl]-2,3-dihydro-1H-benzo[b]xanthen-12yl\}imidoformamide (2c):

Red-green crystals, yield $80 \%, \mathrm{mp} 223-225^{\circ} \mathrm{C}$. ${ }^{1} \mathrm{H}$ NMR $\left(400 \mathrm{MHz}, \mathrm{DMSO}-d_{6}\right), \delta, \mathrm{ppm}(J, \mathrm{~Hz})$ : $9.6(1 \mathrm{H}, \mathrm{s}, \mathrm{CH}=\mathrm{N}), 8.72(2 \mathrm{H}, \mathrm{m}, \mathrm{H}-\mathrm{Ar}), 8.00(1 \mathrm{H}$, $\left.\mathrm{s}, \mathrm{N}=\mathrm{CHN}(\mathrm{Me})_{2}\right), 7.92(1 \mathrm{H}, \mathrm{d}, J=8.30 \mathrm{~Hz}, \mathrm{H} \mathrm{Ar})$, $7.85(1 \mathrm{H}, \mathrm{d}, J=8.30 \mathrm{~Hz}, \mathrm{H} \mathrm{Ar}), 7.77(1 \mathrm{H}, \mathrm{s}, \mathrm{H} \mathrm{Ar})$, $7.65(1 \mathrm{H}, \mathrm{s}, \mathrm{H} \mathrm{Ar}), 7.47(1 \mathrm{H}, \mathrm{t}, J=7.8 \mathrm{~Hz}, \mathrm{H} \mathrm{Ar})$, $7.38(1 \mathrm{H}, \mathrm{t}, J=7.8 \mathrm{~Hz}, \mathrm{H} \mathrm{Ar}), 7.19(1 \mathrm{H}, \mathrm{t}, J=4.88$ $\mathrm{Hz}, \mathrm{H} \mathrm{Ar}), 3.09\left(6 \mathrm{H}, \mathrm{s}, 2 \mathrm{CH}_{3}\right), 2.63-2.60(4 \mathrm{H}, \mathrm{m}$, $\left.2 \mathrm{CH}_{2}\right), 1.71-1.69\left(2 \mathrm{H}, \mathrm{m}, \mathrm{CH}_{2}\right) .{ }^{13} \mathrm{C}$ NMR $(100$ $\left.\mathrm{MHz}, \mathrm{DMSO}-d_{6}\right), \delta$, ppm: $167.4(\underline{\mathrm{CH}}=\mathrm{N}), 160.4$, 158.7, 158.4, 154.8, 149.4, 145.6, 133.8, 129.6, 128.2, 127.0, 126.6, 124.9, 123.2, 121.7, 117.0, 112.8, 110.1, 109.9, 33.9, 24.9, 23.0, 20.6. FT-IR (KBr pellets, $\left.v, \mathrm{~cm}^{-1}\right): 2925$ (aliphatic $\left.\mathrm{C}-\mathrm{H}\right), 1645(\mathrm{C}=\mathrm{N}) . \mathrm{MS}$ (FAB), $m / z\left(I_{\text {rel, }} \%\right): 410[\mathrm{M}+\mathrm{H}]^{+}(100)$. Calculated for $\mathrm{C}_{25} \mathrm{H}_{23} \mathrm{~N}_{3} \mathrm{O}(\%)$ : C, 73.30; $\mathrm{H}, 5.66 ; \mathrm{N}, 17.10$. Found (\%): C, 73.16; H, 5.53; N, 17.22.
$N-(4-\{(E)-[(3-h y d r o x y p h e n y l)$ imino $]$ methyl $\}-$ 2,3-dihydro-1H-benzo[b]xanthen-12-yl)- $N, N$ dimethylimidoformamide (2d):

Red powder, yield $75 \%, \mathrm{mp} 243-244^{\circ} \mathrm{C} .{ }^{1} \mathrm{H}$ NMR (400 MHz, DMSO- $\left.d_{6}\right), \delta$, ppm $(J, \mathrm{~Hz}): 10.40$ $(1 \mathrm{H}, \mathrm{s}, \mathrm{OH}), 8.66(1 \mathrm{H}, \mathrm{s}, \mathrm{CH}=\mathrm{N}), 8.48(1 \mathrm{H}, \mathrm{s}$, $\left.\mathrm{N}=\mathrm{CHN}(\mathrm{Me})_{2}\right), 8.48(1 \mathrm{H}, \mathrm{s}, \mathrm{H} \mathrm{Ar}), 8.27(2 \mathrm{H}, \mathrm{m}$, $\mathrm{H}-\mathrm{Ar}), 8.14(1 \mathrm{H}, \mathrm{d}, J=8.3 \mathrm{~Hz}, \mathrm{H} \mathrm{Ar}), 8.01(1 \mathrm{H}, \mathrm{d}$, $J=8.3 \mathrm{~Hz}, \mathrm{H} \mathrm{Ar}), 7.65(1 \mathrm{H}, \mathrm{t}, J=7.32 \mathrm{~Hz}, \mathrm{H} \mathrm{Ar})$, $7.55(1 \mathrm{H}, \mathrm{t}, J=7.32 \mathrm{~Hz}, \mathrm{H} \mathrm{Ar}), 7.25(1 \mathrm{H}, \mathrm{t}, J=8.3$ $\mathrm{Hz}, \mathrm{H} \mathrm{Ar}), 7.02(1 \mathrm{H}, \mathrm{d}, J=7.81 \mathrm{~Hz}, \mathrm{H} \mathrm{Ar}), 6.65(1$ $\mathrm{H}, \mathrm{d}, J=7.81 \mathrm{~Hz}, \mathrm{H} \mathrm{Ar}), 3.26\left(3 \mathrm{H}, \mathrm{s}, \mathrm{CH}_{3}\right), 3.23$ $\left(3 \mathrm{H}, \mathrm{s}, \mathrm{CH}_{3}\right), 2.73-2.70\left(2 \mathrm{H}, \mathrm{m}, \mathrm{CH}_{2}\right), 2.61-2.59$ $\left(2 \mathrm{H}, \mathrm{m}, \mathrm{CH}_{2}\right), 1.79-1.77\left(2 \mathrm{H}, \mathrm{m}, \mathrm{CH}_{2}\right) \cdot{ }^{13} \mathrm{C} \mathrm{NMR}$ $(100 \mathrm{MHz}, \mathrm{TFA}-d), \delta$, ppm: $170.3(\underline{\mathrm{CH}}=\mathrm{N}), 158.2$ $(\underline{\mathrm{C}}-\mathrm{OH}), 154.8,150.7,140.9,140.8,138.4,134.1$, 133.6, 132.8, 130.9, 130.2, 129.7, 126.9, 126.4, 119.3, $119.1,116.2,111.5,109.2,46.9,39.4,27.1,23.9$, 21.3. FT-IR ( $\mathrm{KBr}$ pellets, $\left.v, \mathrm{~cm}^{-1}\right): 3303(\mathrm{OH})$, 2855-2924 (aliphatic $\mathrm{C}-\mathrm{H}), 1630(\mathrm{C}=\mathrm{N})$. MS $(\mathrm{FAB}), m / z\left(I_{\mathrm{rel},}, \%\right): 424[\mathrm{M}+\mathrm{H}]^{+}(100)$. Calculated for $\mathrm{C}_{27} \mathrm{H}_{25} \mathrm{~N}_{3} \mathrm{O}_{2}(\%)$ : C, 76.57; $\mathrm{H}, 5.95 ; \mathrm{N}, 9.92$. Found (\%): C, 76.44; H, 5.83; N, 9.99.

$N, N-d i m e t h y l-N-(4-\{(E)-[(3-$ nitrophenyl)imino]methyl\}-2,3-dihydro- $1 \mathrm{H}$ benzo[b]xanthen-12-yl)- $N, N$-dimethylimidoformamide (2e):

Light-red powder, yield $65 \%, \mathrm{mp} 251-252^{\circ} \mathrm{C}$. ${ }^{1} \mathrm{H}$ NMR $\left(400 \mathrm{MHz}, \mathrm{DMSO}-d_{6}\right), \delta$, ppm $(J, \mathrm{~Hz})$ : $8.90(1 \mathrm{H}, \mathrm{s}, \mathrm{CH}=\mathrm{N}), 8.02\left(1 \mathrm{H}, \mathrm{s}, \mathrm{N}=\mathrm{CH}-\mathrm{N}(\mathrm{Me})_{2}\right)$, 7.98-7.92 (3H, m, H Ar), 7.83-7.81 $(2 \mathrm{H}, \mathrm{m}, \mathrm{H}$ $\mathrm{Ar}), 7.65-7.72(3 \mathrm{H}, \mathrm{m}, \mathrm{H}-\mathrm{Ar}), 7.47(1 \mathrm{H}, \mathrm{t}, J=8.3$ $\mathrm{Hz}, \mathrm{H} \mathrm{Ar}), 7.39(1 \mathrm{H}, \mathrm{t}, J=8.3 \mathrm{~Hz}, \mathrm{H} \mathrm{Ar}), 3.11(6 \mathrm{H}$, $\left.\mathrm{s}, 2 \mathrm{CH}_{3}\right), 2.61-2.59\left(4 \mathrm{H}, \mathrm{m}, 2 \mathrm{CH}_{2}\right), 1.71-1.69(2 \mathrm{H}$, $\left.\mathrm{m}, \mathrm{CH}_{2}\right) \cdot{ }^{13} \mathrm{C}$ NMR (100 MHz, DMSO- $d_{6}$ TFA- $d$ ), $\delta$, ppm: $167.0(\mathrm{CH}=\mathrm{N}), 160.4,156.9,149.3,148.8$, 141.5, 140.8, 135.3, 131.0, 130.6, 129.5, 129.4, 127.2, $126.9,126.4,122.7,119.5,119.4,112.8,110.9,105.2$, 41.3, 35.3, 24.3, 22.9, 20.4. FT-IR (KBr pellets, $v$, $\left.\mathrm{cm}^{-1}\right): 2931$ (aliphatic $\left.\mathrm{C}-\mathrm{H}\right), 1629(\mathrm{C}=\mathrm{N}), 1530$ (as $\left.\mathrm{NO}_{2}\right), 1341\left(\operatorname{sym} \mathrm{NO}_{2}\right)$. MS (FAB), $m / z\left(I_{\text {rel, }} \%\right)$ : $453[\mathrm{M}+\mathrm{H}]^{+}(100)$. Calculated for $\mathrm{C}_{27} \mathrm{H}_{24} \mathrm{~N}_{4} \mathrm{O}_{3}(\%)$ : $\mathrm{C}, 71.67$; H, 5.35; N, 12.38. Found (\%): C, 71.54; $\mathrm{H}, 5.63 ; \mathrm{N}, 12.49$.

$N, N$-dimethyl-N-(4-\{(Z)-[(2-methyl-5nitrophenyl)imino Jmethyl $\}-2,3-$ dihydro- $1 \mathrm{H}$ benzo[b]xanten-12-yl)imidoformamide (2f):

Orange crystals, yield $63 \%, \mathrm{mp} 236-238^{\circ} \mathrm{C} .{ }^{1} \mathrm{H}$ NMR (400 MHz, DMSO- $\left.d_{6}\right), \delta, \operatorname{ppm}(J, \mathrm{~Hz}): 8.86$ $(1 \mathrm{H}, \mathrm{s}, \mathrm{CH}=\mathrm{N}), 7.93\left(1 \mathrm{H}, \mathrm{s}, \mathrm{N}=\mathrm{CHN}(\mathrm{Me})_{2}\right), 7.90-$ $7.88(2 \mathrm{H}, \mathrm{m}, \mathrm{H}-\mathrm{Ar}), 7.79-7.77(1 \mathrm{H}, \mathrm{m}, \mathrm{H} \mathrm{Ar})$, 7.72-7.71 (2H, m, H Ar), 7.61 (1H, s, H Ar), 7.46$7.36(3 \mathrm{H}, \mathrm{m}, \mathrm{H} \mathrm{Ar}), 3.08\left(6 \mathrm{H}, \mathrm{s}, 2 \mathrm{CH}_{3}\right), 2.65-2.57$ $\left(4 \mathrm{H}, \mathrm{m}, 2 \mathrm{CH}_{2}\right), 2.36\left(3 \mathrm{H}, \mathrm{s}, \mathrm{CH}_{3}\right), 1.71-1.69(2 \mathrm{H}$, 
$\left.\mathrm{m}, \mathrm{CH}_{2}\right) .{ }^{13} \mathrm{C}$ NMR $(100 \mathrm{MHz}, \mathrm{TFA}-d), \delta$, ppm: $167.0(\underline{\mathrm{CH}}=\mathrm{N}), 156.8,149.4,147.0,140.8,139.3$, $135.2,132.3,131.8,130.6,129.4,129.2,128.6,128.3$, 127.3, 126.7, 126.4, 125.9, 123.8, 120.8, 111.0, 105.4, $41.4\left(\mathrm{CH}_{3}\right), 35.4\left(\mathrm{CH}_{3}\right), 24.5,23.0,20.4,18.2\left(\mathrm{CH}_{3}\right)$.

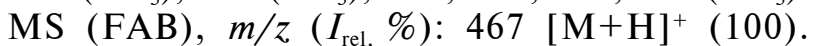
Calculated for $\mathrm{C}_{28} \mathrm{H}_{26} \mathrm{~N}_{4} \mathrm{O}_{3}(\%)$ : C, 72.09; H, 5.62; N, 12.01. Found (\%): C, 72.14; H, 5.73; N, 12.19 . $2-\{[(1 E)-(11-\{[(1 E)-($ dimethylamino $)-$ me thylene ] a mino\}-1,2-dihydrobenzo [g]cyclopenta[b]chromen-3-yl)methylene]amino\}benzoic acid (2g):

Purple powder, yield $73 \%, \operatorname{mp} 241-243^{\circ} \mathrm{C} .{ }^{1} \mathrm{H}$ NMR (400 MHz, TFA- $d$ ), $\delta, \operatorname{ppm}(J, \mathrm{~Hz}): 9.51$ $(1 \mathrm{H}, \mathrm{s}, \mathrm{CH}=\mathrm{N}), 8.88-8.86(2 \mathrm{H}, \mathrm{m}, \mathrm{H} \mathrm{Ar}), 8.58$ $\left(1 \mathrm{H}, \mathrm{s}, \mathrm{N}=\mathrm{CHN}(\mathrm{Me})_{2}\right), 8.44-8.22(6 \mathrm{H}, \mathrm{m}, \mathrm{H} \mathrm{Ar})$, 8.08-7.93 (2H, m, H-Ar), 3.99 (6H, s, 2CH $\mathrm{CH}_{3}$, 3.77$3.75\left(2 \mathrm{H}, \mathrm{m}, \mathrm{CH}_{2}\right), 3.55-3.53\left(2 \mathrm{H}, \mathrm{m}, \mathrm{CH}_{2}\right) .{ }^{13} \mathrm{C}$ NMR (100 MHz, TFA-d), $\delta$, ppm: $176.9\left(\mathrm{CO}_{2} \mathrm{H}\right)$, $171.6(\underline{\mathrm{CH}}=\mathrm{N}), 156.9,151.3,147.8,137.8,135.7$, 133.6, 132.7, 132.5, 130.7, 130.3, 129.6, 125.9, 117.0, 46.9, 39.9, 25.9, 24.9. MS (FAB), $m / z\left(I_{\text {rel, }} \%\right): 438$ $[\mathrm{M}+\mathrm{H}]^{+}$(32). Calculated for $\mathrm{C}_{27} \mathrm{H}_{23} \mathrm{~N}_{3} \mathrm{O}_{3}(\%)$ : C, 74.12; H, 5.30; N, 9.60. Found (\%): C, 74.22; H, 5.34; N, 9.46.

\section{REFERENCES}

1. Patil S., Jadhav S.D., Patil U. Natural acid catalyzed synthesis of Schiff base under solvent-free condition: as a green approach // Arch. Appl. Sci. Res. - 2012. - Vol.4. - No. 2. P.1074-1078.

2. Novel one-pot synthesis of Schiff base compounds derived from different diamine \& aromatic aldehyde catalyzed by $\mathrm{P}_{2} \mathrm{O}_{5} / \mathrm{SiO}_{2}$ under free-solvent condition at room temperature / S.M. Devidas, S.H. Quadri, S.A. Kamble, F.M. Syed, D.Y. Vyavhare // J. Chem. Pharm. Res. - 2011. - Vol.3. - No. 2. - P.489-495.

3. A new soluble Schiff base polymer with a double azomethine group synthesized by oxidative polycondensation / A. Ozbulbul, H. Mart, M. Tuncel, S. Serin // Des. Monom. Polym. - 2006. - Vol.9. - No. 2. - P.169-179.

4. The role of a Schiff base scaffold, N-(2-hydroxy acetophenone) glycinate-in overcoming multidrug resistance in cancer / A. Ganguly, P. Chakraborty, K. Banerjee, S.K. Choudhuri // Eur. J. Pharm. Sci. - 2014. - Vol.51. - P.96-109.

5. Resistance-switchable graphene oxide-polymer nanocomposites for molecular electronics / G. Liu, Y. Chen, R.-W. Li, B. Zhang, E.-T. Kang, C. Wang, X.Zhuang // ChemElectroChem. - 2014. - Vol.1. - No. 3. - P.514-519.

6. Through-resonance assisted ionic hydrogen bonding in 5-nitro-N-salicylideneethylamine / T.M. Krygowski, K. Wozniak, R. Anulewicz, D. Pawlak, W. Kolodziejski, E. Grech, A. Szady /
/ J. Phys. Chem. A. - 1997. - Vol.101. - P.9399-9404.

7. Овденко В.Н., Сыромятников В.Г., Колендо А.Ю. Полиазометины: синтез, свойства и применение (обзор) // Полимерные материалы и технологии. - 2017. - Т.4. - № 1. - C.6-31.

8. Synthesis and characterization of some chelate polymers of polySchiff base ligand / G.B. Pethe, A.R. Yaul, J.B. Devhade, A.S. Aswar // Der Pharma Chemica - 2010. - Vol.2. - No. 3. P.301-308.

9. Xavier A., Srividhya N. Synthesis and study of Schiff base ligands // IOSR Journal of Applied Chemistry. - 2014. Vol.7. - No. 11. - P.6-15.

10. All-aromatic liquid crystal triphenylamine-based poly(azomethine)s as hole transport materials for opto-electronic applications / J.C. Hindson, B. Ulgut, R.H. Friend, N.C. Greenham, B. Norder, A. Kotlewskic, T.J. Dingemans // J. Mater. Chem. - 2010. - Vol.20. - P.937-944.

11. Rearrangement of $5^{\prime}, 6^{\prime}, 7^{\prime}, 8^{\prime}$-tetrahydro-1'Hspiro(cyclohexane-1,2'-quinazolin)-4'(3'H)-one during Vilsmeier reaction / V.I Markov, O.K. Farat, S.A. Varenichenko, E.V. Velikaya // Mendeleev Commun. - 2012. - Vol.22. - P.101102.

12. Synthesis and formylation of substituted 2-spiropyrimidin-4-ones and related compounds / V.I. Markov, O.K. Farat, S.A. Varenichenko, E.V. Velikaya, R.I. Zubatyuk, O.V. Shishkin // Chem. Heterocycl. Compd. - 2013. - Vol.49. - P.1158-1165.

13. The interaction of 4,5-diformyl-2,3,6,7,8,10hexahydroacridine-8a( $1 H)$-carbonitrile with $\mathrm{N}$-nucleophiles / E.V. Zaliznaya, T.P. Polishuk, S.A. Varenichenko, O.K. Farat, V.I. Markov // J. Org. Pharm. Chem. - 2016. - Vol.14. - P.3842.

14. Варениченко С.А., Зализная Е.В., Марков В.И. Реакционная способность 4-метил-1,5-диазаспиро[5.5]ундек-3ен-2-она и его производных // Вопросы химии и химической технологии. -2017 . - № 4. - С.8-13.

15. Vilsmeier-Haack reagent: an eficient reagent for the transformation of substituted 1,3-naphthoxazines into xanthenetype dyes / O.K. Farat, I.V. Ananyev, S.A. Varenichenko, A.L. Tatarets, V.I. Markov // Tetrahedron. - 2019. - Vol.75. No. 19. - P.2832-2842.

Received 27.05.2019

\section{СИНТЕЗ НОВИХ ОСНОВ ШИФФА НА ОСНОВІ ФОРМІЛПОХІДНИХ КСАНТЕНІВ}

\section{С.А. Варениченко, О.К. Фарат, А.В. Мазепа, В.И. Марков}

У результаті реакції тетрациклічних формілпохідних ксантенів з ароматичними амінами були одержані нові основи Шиффа в присутності пари крапель етанової кислоти. Структури цих сполук були досліджені методами ІЧ, ЯМР і масспектрометрії. Формілксантени продемонстрували поглинання і випромінення в діапазоні 424-435 нм і 524-559 нм, відповідно, в розчинниках різної полярності. Вихідні формілксантени характеризувалися досить великим Стоксовим зсувом 100- 
130 нм і помірним квантовим виходом флуоресценції. Одержані основи Шиффа не виявляють флуоресценції, за винятком двох продуктів з антраніловою кислотою. Ці сполуки мають слабко структуровані спектри поглинання з максимумами при 527 i 532 нм. Максимуми люмінесценції були виявлені в діапазоні 582 і 598 нм, в результаті одержані сполуки характеризуються низьким квантовим виходом флуоресценції в діапазоні 0,01-0,02 в розчині ацетонітрилу і Стоксовим зсувом 55 і 65 нм.

Ключові слова: форміл ксантени, основи Шиффа, ароматичні аміни, флуоресценція, Стоксів зсув.

\section{SYNTHESIS OF NEW SCHIFF BASES BASED ON FORMYL DERIVATIVES OF XANTHENES}

S.A. Varenichenko ${ }^{a}$, ", O.K. Farat ${ }^{a}$, A.V. Mazepa ${ }^{b}$, V.I. Markov ${ }^{a}$ a Ukrainian State University of Chemical Technology, Dnipro, Ukraine

${ }^{b}$ A.V. Bogatsky Physico-Chemical Institute of the National Academy of Sciences of Ukraine, Odessa, Ukraine

*e-mail: svetlanavarenichenko@gmail.com

New Schiff bases were synthesized by the reaction of tetracyclic formyl derivatives of xanthenes with aromatic amines in the presence of a vapor of acetic acid drops. The structures of these compounds were investigated by FTIR, NMR spectroscopies and massspectrometric technique. Formyl xanthenes showed absorption and emission in the range of 424-435 $\mathrm{nm}$ and $524-559 \mathrm{~nm}$, respectively, in solvents with different polarities. Initial formyl xanthenes were characterized by a suficiently high Stokes shift of 100-130 nm and a moderate fluorescence quantum yield. The prepared Schiff bases exhibit no fluorescence, with the exception of two products with antranilic acid. These compounds show weakly structured absorption spectra with maxima at $527 \mathrm{~nm}$ and $532 \mathrm{~nm}$. Luminescence maxima were found in the range of 582 and $598 \mathrm{~nm}$; as a result, the prepared compounds are characterized by low fluorescence quantum yields in the range of 0.01-0.02 in acetonitrile solution and the Stokes shift of $55 \mathrm{~nm}$ and $65 \mathrm{~nm}$.

Keywords: formyl xanthenes; Schiff bases; aromatic amines; fluorescence; Stokes shift.

\section{REFERENCES}

1. Patil S., Jadhav S.D., Patil U. Natural acid catalyzed synthesis of Schiff base under solvent-free condition: as a green approach. Archives of Applied Science Research, 2012, vol. 4, no. 2, pp. 1074-1078.

2. Devidas S.M., Quadri S.H., Kamble S.A., Syed F.M., Vyavhare D.Y. Novel one-pot synthesis of Schiff base compounds derived from different diamine \& aromatic aldehyde catalyzed by $\mathrm{P}_{2} \mathrm{O}_{5} / \mathrm{SiO}_{2}$ under free-solvent condition at room temperature. Journal of Chemical and Pharmaceutical Research, 2011, vol. 3, no. 2, pp. 489-495.

3. Ozbulbul A., Mart H., Tuncel M., Serin S. A new soluble Schiff base polymer with a double azomethine group synthesized by oxidative polycondensation. Designed Monomers and Polymers, 2006, vol. 9, pp. 169-179.

4. Ganguly A., Chakraborty P., Banerjee K., Choudhuri S.K. The role of a Schiff base scaffold, N-(2-hydroxy acetophenone) glycinate-in overcoming multidrug resistance in cancer. European Journal of Pharmaceutical Sciences, 2014, vol. 51, pp. 96-109.
5. Liu G., Chen Y., Li R.-W., Zhang B., Kang E.-T., Wang C., Zhuang X. Resistance-switchable graphene oxidepolymer nanocomposites for molecular electronics. ChemElectro Chem, 2014, vol. 1, pp. 514-519.

6. Krygowski T.M., Wozniak K., Anulewicz R., Pawlak D., Kolodziejski W., Grech E., Szady A. Through-resonance assisted ionic hydrogen bonding in 5-nitro- $\mathrm{N}$-salicylideneethylamine. Journal of Physical Chemistry A, 1997, vol. 101, pp. 9399-9404.

7. Ovdenko V.N., Syromyatnikov V.G., Kolendo A.Yu. Poliazometiny: sintez, svoistva i primenenie (obzor) [Polyazomethins: synthesis, properties and applications (a review)]. Polimernye Materialy i Tekhnologii, 2017, vol. 4, no. 1, pp. 6-31. (in Russian).

8. Pethe G.B., Yaul A.R., Devhade J.B., Aswar A.S. Synthesis and characterization of some chelate polymers of polySchiff base ligand. Der Pharma Chemica, 2010, vol. 2, no. 3, pp. 301-308.

9. Xavier A., Srividhya N. Synthesis and study of Schiff base ligands. IOSR Journal of Applied Chemistry, 2014, vol. 7, no. 11, pp. 6-15.

10. Hindson J.C., Ulgut B., Friend R.H., Greenham N.C., Norder B., Kotlewskic A., Dingemans T.J. All-aromatic liquid crystal triphenylamine-based poly(azomethine)s as hole transport materials for opto-electronic applications. Journal of Materials Chemistry, 2010, vol. 20, pp. 937-944.

11. Markov V.I., Farat O.K., Varenichenko S.A., Velikaya E.V. Rearrangement of $5^{\prime}, 6^{\prime}, 7^{\prime}, 8^{\prime}$-tetrahydro-1" $\mathrm{H}$ spiro(cyclohexane-1,2'-quinazolin)-4'(3"H)-one during Vilsmeier reaction. Mendeleev Communications, 2012, vol. 22, pp. 101-102.

12. Markov V.I., Farat O.K., Varenichenko S.A., Velikaya E.V., Zubatyuk R.I., Shishkin O.V. Synthesis and formylation of substituted 2-spiropyrimidin-4-ones and related compounds. Chemistry of Heterocyclic Compounds, 2013, vol. 49, pp. $1158-1165$.

13. Zaliznaya E.V., Polishuk T.P., Varenichenko S.A., Farat O.K., Markov V.I. The interaction of 4,5-diformyl2,3,6,7,8,10-hexahydroacridine- $8 \mathrm{a}(1 \mathrm{H})$-carbonitrile with N-nucleophiles. Journal of Organic Pharmaceutical Chemistry, 2016, vol. 14, pp. 38-42.

14. Varenichenko S.A., Zalizna E.V., Markov V.I. Reaktsionnaya sposobnost' 4-metil-1,5-diazaspiro[5.5]undek-3en-2-ona i ego proizvodnykh [Reactivity of 4-methyl-1,5diazaspiro[5.5] undec-3-en-2-one and its derivatives]. Voprosy Khimii i Khimicheskoi Tekhnologii, 2017, no. 4, pp. 8-13. (in Russian).

15. Farat O.K., Ananyev I.V., Varenichenko S.A., Tatarets A.L., Markov V.I. Vilsmeier-Haack reagent: an efficient reagent for the transformation of substituted 1,3-naphthoxazines into xanthene-type dyes. Tetrahedron, 2019, vol. 75, pp. 2832-2842. 